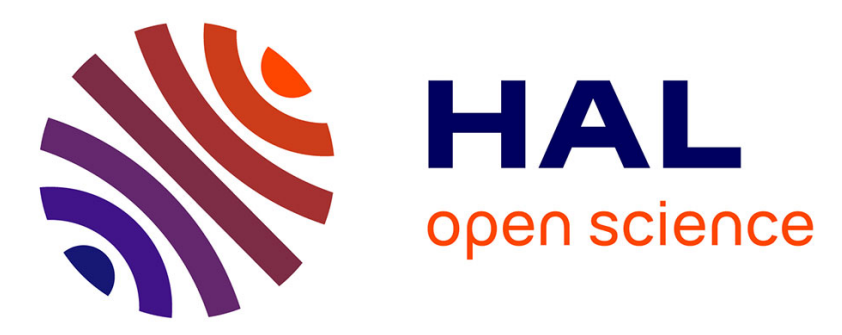

\title{
Atomic Structure of Glassy GeTe 4 as a Playground to Assess the Performances of Density Functional Schemes Accounting for Dispersion Forces
}

Pier Luigi Silvestrelli, Évelyne Martin, Mauro Boero, Assil Bouzid, Guido Ori, Carlo Massobrio

\section{To cite this version:}

Pier Luigi Silvestrelli, Évelyne Martin, Mauro Boero, Assil Bouzid, Guido Ori, et al.. Atomic Structure of Glassy GeTe 4 as a Playground to Assess the Performances of Density Functional Schemes Accounting for Dispersion Forces. Journal of Physical Chemistry B, 2020, 124 (49), pp.11273-11279. 10.1021/acs.jpcb.0c08628 . hal-03086320

\section{HAL Id: hal-03086320 https://hal.science/hal-03086320}

Submitted on 22 Dec 2020

HAL is a multi-disciplinary open access archive for the deposit and dissemination of scientific research documents, whether they are published or not. The documents may come from teaching and research institutions in France or abroad, or from public or private research centers.
L'archive ouverte pluridisciplinaire HAL, est destinée au dépôt et à la diffusion de documents scientifiques de niveau recherche, publiés ou non, émanant des établissements d'enseignement et de recherche français ou étrangers, des laboratoires publics ou privés. 


\title{
The atomic structure of glassy $\mathrm{GeTe}_{4}$ as a
}

\section{playground to assess the performances of density}

\section{functional schemes accounting for dispersion forces}

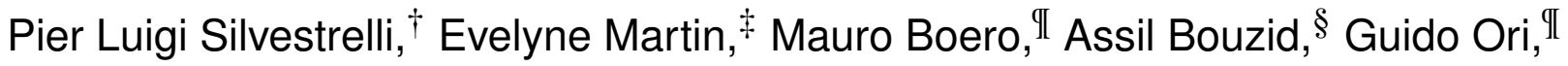 \\ and Carlo Massobrio*,II
}

$\dagger$ †ipartimento di Fisica e Astronomia "G. Galilei," Universitá di Padova, via Marzolo 8, I-35131

Padova, Italy and CNR-IOM Democritos, via Bonomea 265, I-34136 Trieste, Italy

†Université de Lille, CNRS, Centrale Lille, ISEN, Université Valenciennes, UMR 8520-IEMN,

$$
\text { F-59000 Lille, France }
$$

IUniversity of Strasbourg, Institut de Physique et Chimie des Matériaux de Strasbourg, CNRS, UMR 7504, 23 rue du Loess, F-67034 France

§Institut de Recherche sur les Céramiques, UMR 7315 CNRS-Université de Limoges, Centre Européen de la Céramique, 12 rue Atlantis 87068 Limoges, France

\section{E-mail: carlo.massobrio@ipcms.unistra.fr}

\begin{abstract}
The atomic structure of glassy $\mathrm{GeTe}_{4}$ is obtained in the framework of first-principles molecular dynamics by considering five different approaches for the description of the electronic structure within density functional theory. Among these schemes, one is not corrected by the account of the dispersion forces and it is based on the BLYP exchange correlation (XC) functional, while all the others are intended to account for the dispersion forces according to different theoretical strategies. In particular, by maintaining the BLYP expression for the
\end{abstract}


XC functional, two of them (BLYP-D2 and BLYP-D3) exploit the Grimme expressions for the dispersion forces, while the fourth scheme is based on the Maximally Localized Wannier functions (MLWF). Finally, we also considered the rVV10 functional constructed to include seamlessly the dispersion part. Our results point out the better performances of the BLYP-D3 and MLWF in terms of comparison with experimental data for the total pair correlation functions, BLYP-D2 and rVV10 being closer to the uncorrected BLYP data. The implications of such findings are discussed by considering the overall limited impact of dispersion forces on the atomic structure of glassy $\mathrm{GeTe}_{4}$.

\section{Introduction}

Two main reasons are at origin of the widespread interest in disordered network forming materials containing Ge-Te bonded structural units. The first is deeply rooted into the role they play as representative systems in phase change materials (PCMs) or in devices prone to applications for optics and far infrared transmission ${ }^{1,2}$. These latter exhibit enhanced glass stability as an essential prerequisite while PCMs are typically poor glass formers due to their inherent structural instability ${ }^{3}$. The second reason is more fundamental, since chalcogenide disordered networks are an excellent playground for the assessment of bonding properties escaping any clear-cut classification in terms of standard ionic or covalent bonding. This limitation has been somewhat overcome by invoking the notion of iono-covalent bonding to describe localized distribution of charges around the atomic sites coexisting with highly directional bonding ${ }^{4-6}$. However, the search of a quantitative theoretical description remains challenging. The case of Ge-Te glasses is even more elusive. These systems are not only highly sensitive, within density functional theory (DFT), to the choice of the exchange-correlation (XC) functional but also, for specific concentrations, to the neglect or the account of dispersion forces. In a paper devoted to glassy $\mathrm{GeTe}_{4}$, we pointed out that its structure depends on the combined choice of the XC functional and of dispersion (van der Waals, vdW) corrections ${ }^{6}$. This work was carried out by relying on the first-principles molecular dynamics (FPMD) plane waves DFT scheme. In particular, it was demonstrated that the impact of vdW forces is not 
as crucial as the optimal choice of the $\mathrm{XC}$ functional (BLYP ${ }^{7,8}$ turning out to be the preferred one) but it cannot be neglected a priori as it occurs in the original Kohn-Sham DFT expression.

Such conclusion was reached via the application of the vdW scheme due to Grimme $\left(\mathrm{vdW}_{\mathrm{G}}\right)$, in the version named $\mathrm{D} 2{ }^{9}$. A further set of calculations on glassy $\mathrm{GeTe}_{4}$ was motivated by two considerations $^{10}$. First, it appeared that a single dispersion scheme cannot be taken as a universal recipe equally effective for any system, this calling for the account of at least one alternative choice to compare with. Second, there is some concern on the neglect, in D2-Grimme, of updates in the dispersion coefficients resulting from the evolution in time of the electronic structure, closer in spirit to the combined dynamical evolution of ionic and electronic degrees of freedom occurring in first-principles molecular dynamics (FPMD) ${ }^{11}$. Accordingly, in Ref. 10, we focused on the comparison between distinct sets of BLYP data, namely the two already available (not including dispersion forces, named NovdW and adopting the D2-Grimme scheme, named vdW $\mathrm{W}_{\mathrm{G}}$ ) and a third one, making use of the maximally localized Wannier functions (MLWF) framework (vdWW hereafter), known for exploiting the evolution of the electronic structure when updating the dispersion coefficients $^{12-15}$. Taken altogether, the results obtained did not provide unambiguous indications. In fact, the vdW strategy produced pair correlation functions agreeing well with the NovdW set of data but differing from those obtained via $\mathrm{vdW}_{\mathrm{G}}$. In turn, $\mathrm{vdW}_{\mathrm{G}}$ data were in better agreement with the experimental counterpart. These pieces of evidence call for a more complete analysis, extended to other vdW schemes, in order to achieve the following goals: $a$ ) a more robust conclusion on the impact of the dispersion forces, $b$ ) the assessment of the actual improvements induced by dispersion coefficients adapting to changes in the electronic structure with respect to more empirical ones and, $c$ ) some hints on the overall capability of the correction schemes not to modify artificially the structural data for bonding situations not crucially demanding their explicit account. This latter point is likely to be quite subtle since one would like to estimate correctly dispersion forces regardless of their being a priori of limited impact. For instance, in the case of glassy $\mathrm{GeTe}_{4}$, plausible physico-chemical arguments (i.e. the presence of predominant iono-covalent interactions) indicate that they should not affect substantially structural properties. 
In this paper atomic-scale modelling of glassy $\mathrm{GeTe}_{4}$ is carried out within DFT-FPMD by performing extensive calculations with five different approaches for the description of the dispersion forces. This new insight considerably improves upon the preliminary report of Ref. 10. We have accounted not only for two additional vdW schemes but we have also produced new time trajectories to reach a total number of 11 independent ones. As a result, we have gathered more compelling information on the changes induced to the network topology by a variety of different treatments of the long range van des Waals interactions.

This paper is organized as follows. The introduction is followed by section 2 describing our methods of calculation, comprising the theoretical framework and the technical details of the FPMD simulations as well as the different schemes employed to account for the dispersion forces. Section 3 contains in two different subsections results for the total and the partial pair correlation functions. Conclusive remarks are collected in section 4 .

\section{Theoretical Methods}

In order to fully appreciate the insight provided by the present investigation and the underlying methodology, we recall the computational framework employed in Ref. 10. Glassy GeTe 4 was made of 215 atoms (43 Ge, $172 \mathrm{Te}$ ) in a supercell of cubic shape with edge $19.24 \AA$. Such system was studied within the Car-Parrinello ${ }^{11}$ molecular dynamics (CPMD) scheme as implemented

in the CPMD code ${ }^{16}$, by adopting the exchange functional by Becke ${ }^{7}$ and the correlation functional by Lee, Yang and Parr $^{8}$ (BLYP). An energy cutoff of 40 Ry was employed to account for valence electrons within a plane wave basis set, by restricting the Brillouin zone integration to the $\Gamma$ point ${ }^{6,17,18}$. Other relevant features were the control of the temperatures of the ions and of the fictitious electronic degrees of freedom via Nosé-Hoover thermostats ${ }^{19-21}$ and the choice of an integration step of 5 a.u. (0.121 fs). The Nosé-Hoover chain consisted of two thermostats, characterized by an oscillation frequency of 6 and $60 \mathrm{THz}$, respectively.

In regard to the choice of the BLYP exchange-correlation functional, this is rooted into the 
better performances exhibited by this recipe for disordered Ge-based chalcogenides ${ }^{22,23}$. In particular, one obtains an improved description of the short range Ge environment corresponding to an enhanced localized behavior of the electron density. Such effect is predominant over electronic delocalization that favors the metallic character, a drawback inherent in generalized gradient approximation approaches based on the uniform electron gas as reference system (as the one due to the Perdew and Wang ${ }^{24}$.

Dispersion forces were included by using either the DFT-D2 formula devised by Grimme ${ }^{9}$ or by resorting to a recipe (refered to hereafter as $\mathrm{vdW}_{\mathrm{W}}$ ) based on the concept of the maximally localized Wannier functions (MLWFs) ${ }^{12-15}$. Briefly, the vdW $\mathrm{W}_{\mathrm{W}}$ scheme can be described by relying on the following relationships, the first containing the $C_{6, n l}$ coefficients ${ }^{25}$

$$
E_{v d W}=-\sum_{n<l} f\left(r_{n l}\right) \frac{C_{6, n l}}{r_{n l}^{6}}
$$

(in which the subscripts $n$ and $l$ run over all atoms or fragments of the system and $f\left(r_{n l}\right)$ is a damping function avoiding unphysical divergence and double counting of correlations at short range) and the second expressing them in terms of the MLWFs $w_{n}(\mathbf{r})$ as follows

$$
C_{6, n l}=\frac{3}{32 \pi^{3 / 2}} \int_{r \leq r_{\text {cut }}} \int_{r^{\prime} \leq r_{\text {cut }}} \frac{w_{n}(\mathbf{r}) \cdot w_{l}\left(\mathbf{r}^{\prime}\right)}{w_{n}(\mathbf{r})+w_{l}\left(\mathbf{r}^{\prime}\right)} d^{3} r^{\prime} d^{3} r .
$$

The MLWFs approach allows for an intuitive interpretation of the bonding properties of condensedmatter systems and are at the heart of the modern theory of polarization ${ }^{26,27}$. In principle, this schema is very well suited to provide additional information on the electronic structure characterizing the different realizations of glassy $\mathrm{GeTe}_{4}$. However, such an extension is outside the scope of 
the present study focused on the correlation between structural properties and different vdW correction to the Kohn-Sham DFT energy functional. Van der Waals-corrected DFT approaches based on MLWFs proved to be successful in describing many systems: small molecules, water clusters, graphite and graphene, water layers interacting with graphite, interfacial water on semiconducting substrates, hydrogenated carbon nanotubes, molecular solids, the interaction of rare gases and small molecules with metal surfaces,... (see, for instance, Ref. 28 and references therein). The MLWFs approach performs well since it combines the simplicity of the semiempirical formalism with the accuracy of the first principles approaches. In fact, being based on localized electronic orbitals, they are well suited to describe small electronic effects following configuration changes, thereby largely increasing the transferability of the approach (for instance, charge polarization effects are naturally included).

In the present study, the set of methods to account for dispersion forces is completed by considering two additional theoretical schemes. We also resorted to a computational package other than CPMD. More explicitly, FPMD calculations have been performed in the framework of the Quantum Espresso package ${ }^{29-31}$ by selecting either the Car Parrinello ${ }^{11}$ or the Born-Oppenheimer (BO) scheme. This latter amounts to a search of the electronic ground state for each ionic configuration and is characterized by an integration step of 50 a.u. (1.21 fs).

We have increased the statistical sampling of our analysis by taking advantage of new trajectories produced for the same system at room temperature, thereby allowing for averages to be taken by considering previous and new results. As in Ref. 10, trajectories were produced for the BLYP (NovdW), BLYP-D2 (vdW $\mathrm{D}_{\mathrm{D}}$ herafter) and MLWF ( $\left.\mathrm{vdW}_{\mathrm{W}}\right)$ cases. In addition, we also considered the Grimme D3 expression ( $\mathrm{vdW}_{\mathrm{D} 3}$ herafter) expected to ensure improved accuracy and range of applicability with respect to $\mathrm{D} 2^{32}$. In view of its character of exchange-correlation functional constructed to include seamlessly the dispersion part, our study has been also extended to FPMD trajectories produced by using the rVV10 functional $\left(\mathrm{vdW}_{\mathrm{VV}}\right)^{33,34}$. In fact, unlike the Grimme and MLWF methods, rVV10 (as its pristine counterpart VV10 ${ }^{35}$ ) does not feature any separation between the DFT Kohn-Sham functional and the dispersion part. This makes its use particularly 
appealing in the context of a thorough analysis intended to assess the impact of vdW forces on a prototypical disordered chalcogenide. Overall, the theoretical tools employed in Ref. 10 and in the present work can be grouped into three categories: those accounting for dispersion forces via coefficients reflecting (MLWF) $(a)$ or not reflecting (BLYP-D2 and BLYP-D3) $(b)$ changes in the electronic structure during the motion and $(c)$ the rVV10 method built as a functional including dispersion forces via a non-local construction with no analytical separation from the energy functional.

A summary of the different combinations of DFT schemes together with the way FPMD has been implemented (CP or BO) is given in Table 1.

Table 1: DFT schemes and FPMD methodologies employed in this work and in Ref. 10. CP stands for Car-Parrinello and BO for Born-Oppenheimer first principles molecular dynamics

\begin{tabular}{|c|c|c|}
\hline $\mathrm{CP}^{10}$ & $\mathrm{CP}$ & BO \\
\hline NovdW (BLYP) & NovdW(BLYP) & NovdW(BLYP) \\
\hline $\mathrm{vdW}_{\mathrm{D} 2}($ Grimme-D2) & $\mathrm{vdW}_{\mathrm{D} 2}($ Grimme-D2) & $\mathrm{vdW}_{\mathrm{D} 2}($ Grimme-D2) \\
\hline $\mathrm{vdW}_{\mathrm{W}}(\mathrm{BLYP}-\mathrm{MLWF})$ & $\mathrm{vdW}_{\mathrm{VV}}(\mathrm{rVV} 10)$ & $\mathrm{vdW}_{\mathrm{D} 3}($ Grimme-D3) \\
\hline & $\mathrm{vdW}_{\mathrm{W}}(\mathrm{BLYP}-\mathrm{MLWF})$ & $\mathrm{vdW}_{\mathrm{VV}}(\mathrm{rVV} 10)$ \\
\hline
\end{tabular}

The first column refers to Ref. 10. First of all, the same heating and cooling process are used to produce a second $\mathrm{MLWF}\left(\mathrm{vdW}_{\mathrm{W}}\right)$ simulation run. Concerning the production of the other trajectories, one configuration at $T=300 \mathrm{~K}$ among those exploited in Ref. 10 is taken as the initial one to initiate two kinds of thermal cycles. In the first (CPMD method) heating has occurred up to a temperature $T=900 \mathrm{~K}$ over $15 \mathrm{ps}$ to allow for significant diffusion, followed by a rapid quench (in $4 \mathrm{ps)} \mathrm{to} T=300 \mathrm{~K}$ with statistical averages calculated over $6 \mathrm{ps}$ at this same temperature. In the second (BO method) one of the previous sets of coordinates at $T=900 \mathrm{~K}$ is selected to start a cooling process lasting $50 \mathrm{ps}$ down to $T=300 \mathrm{~K}$, followed by data collection over a further trajectory of $20 \mathrm{ps}$ at the same temperature. BLYP-D3 results are available within the FPMD-BO calculations only.

The availability of a distinct number of equilibrium trajectories (three, two or a single one) among the different choices for the dispersion forces does not affect the essence of our results. Indeed, for a specific case, deviations from the mean found when several trajectories are available 
are smaller than the differences occurring among the different schemes.

\section{Results and Discussion}

\subsection{Total pair correlation functions.}

In what follows, we concentrate on the total pair correlation function $g_{\mathrm{T}}(r)$ obtained as a weighted linear combination of the three partial pair correlation functions $g_{\alpha \beta}(r)$, where $\alpha$ and $\beta$ denote the chemical species considered (Ge, Te). The focus will be on a comparative analysis of the pair correlation functions obtained in the absence (NovdW, BLYP data) and in the presence of dispersion forces, these latter expressed in four different forms $\left(\mathrm{vdW}_{\mathrm{D} 2}, \mathrm{BLYP}-\mathrm{D} 2, \mathrm{vdW}_{\mathrm{D} 3}, \mathrm{BLYP}-\mathrm{D} 3\right.$, $\mathrm{vdW}_{\mathrm{W}}$, BLYP-MLWF and vdWvV, rVV10).

As a prerequisite, it is instructive to define under which conditions the performances of the different vdW schemes will be examined. Having in mind the impact of the dispersion forces on the structural properties, we choose not to account for short range contributions corresponding to distances up to the first minimum of the pair correlation function, say smaller than $3 \AA$. For this reason, there is little that can be added to the fact that the depth of the first minimum observed experimentally is not entirely reproduced by any of schemes we implemented (see Fig. 1). Overall, quantitative comparisons and conclusions drawn thereof will not depend on the degree of variability found in the height and shape of the first peaks in the pair correlation functions, clearly not directly sensitive to the action of the dispersion forces. Variations of this specific feature can be mostly ascribed to statistical fluctuations affecting, in the first place, Ge-Ge correlations, the number of Ge atoms being limited to 43 in our simulations. This is why our set of comparisons are based most exclusively on the behaviors recorded for distances larger than $3 \AA$.

For $g_{\mathrm{T}}(r)$ we provide a direct comparison among all cases and also the function $\Delta$ (exp,tot) that results from the difference between the various $g_{\mathrm{T}}(r)$ and the experimental data. Analogously, $\Delta($ NovdW, tot $)$ is the difference between all calculated $g_{\mathrm{T}}(r)$ and the NovdW counterpart. Finally, the $\Delta$ functions are exploited to calculate the integrals of their absolute values on the range $[3-9]$ 
$\AA$ such quantities being inversely proportional to the agreement between two given curves. The case of $g_{\mathrm{T}}(r)$ (Fig. 1) is indicative of small differences between the five different schemes we considered.

One notes that $\mathrm{vdW}_{\mathrm{D} 3}$ improves considerably upon $\mathrm{vdW}_{\mathrm{D} 2}$, both of them performing better than

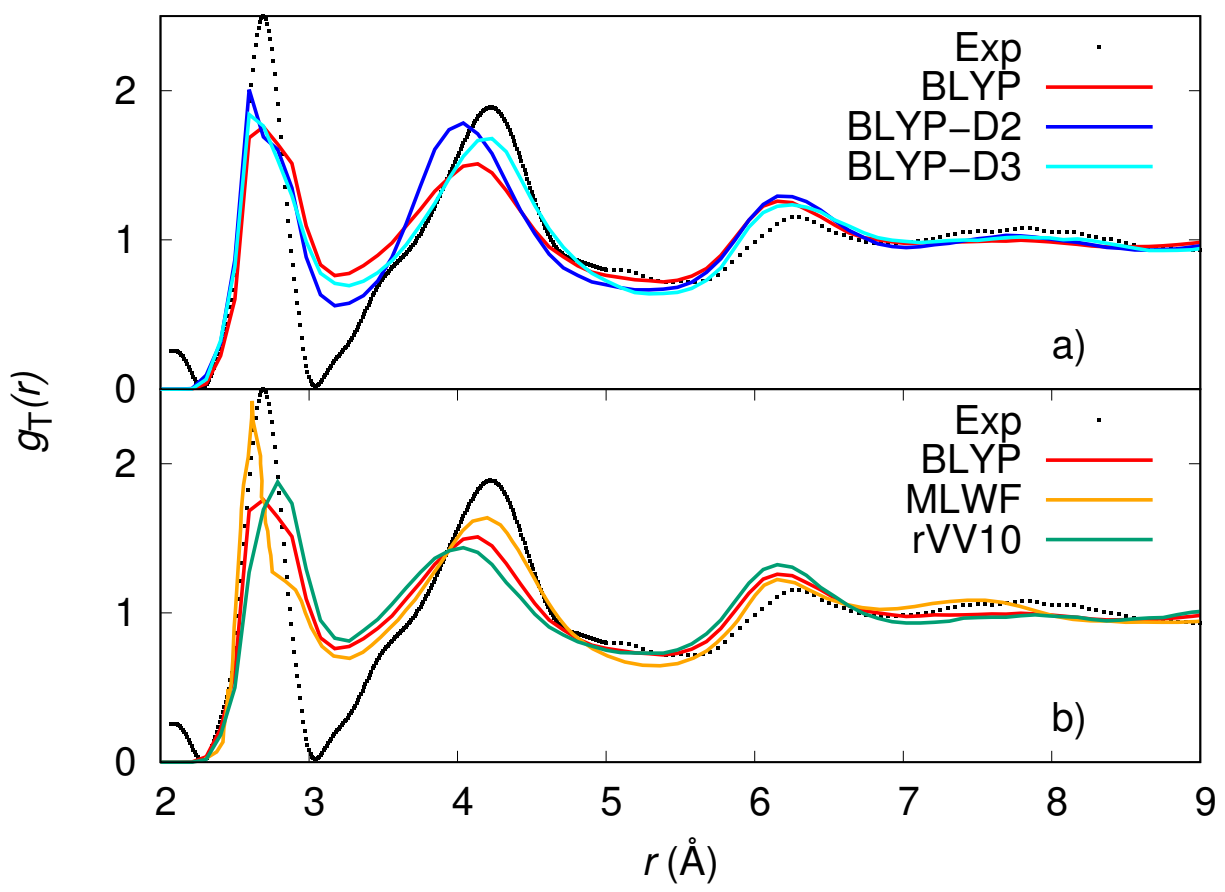

Figure 1: $g_{\mathrm{T}}(r)$. Comparing the five different schemes employed to account for dispersion forces with experiments (from Ref. 36) in the case of the total pair correlation function.

the NovdW scheme. Interestingly, $\mathrm{vdW}_{\mathrm{W}}$ is also closer to experiments than NovdW while $\mathrm{vdW}_{\mathrm{VV}}$ is the farthest from the experimental data. A quantitative summary of this analysis is presented in Table 2 with further evidence given in Fig. 2 ( $\Delta$ (exp,tot) function).

Table 2: Integral of the absolute value of the differences between a given set of data of $g_{\mathrm{T}}(r)$ and the experimental counterpart on the range $[3-9] \AA$.

\begin{tabular}{|c|c|}
\hline Functional & $\mathrm{I}_{\mid \Delta(\text { exp,tot }) \mid}$ (a. u. $)$ \\
\hline BLYP-D3 (vdW & 1.044 \\
\hline BLYP-MLWF (vdW $)_{W}$ & 1.125 \\
\hline BLYP-D2 (vdW & 1.397 \\
\hline BLYP (NowdW) & 1.439 \\
\hline $\mathrm{rVV} 10(\mathrm{vdW} \mathrm{VV})$ & 1.864 \\
\hline
\end{tabular}




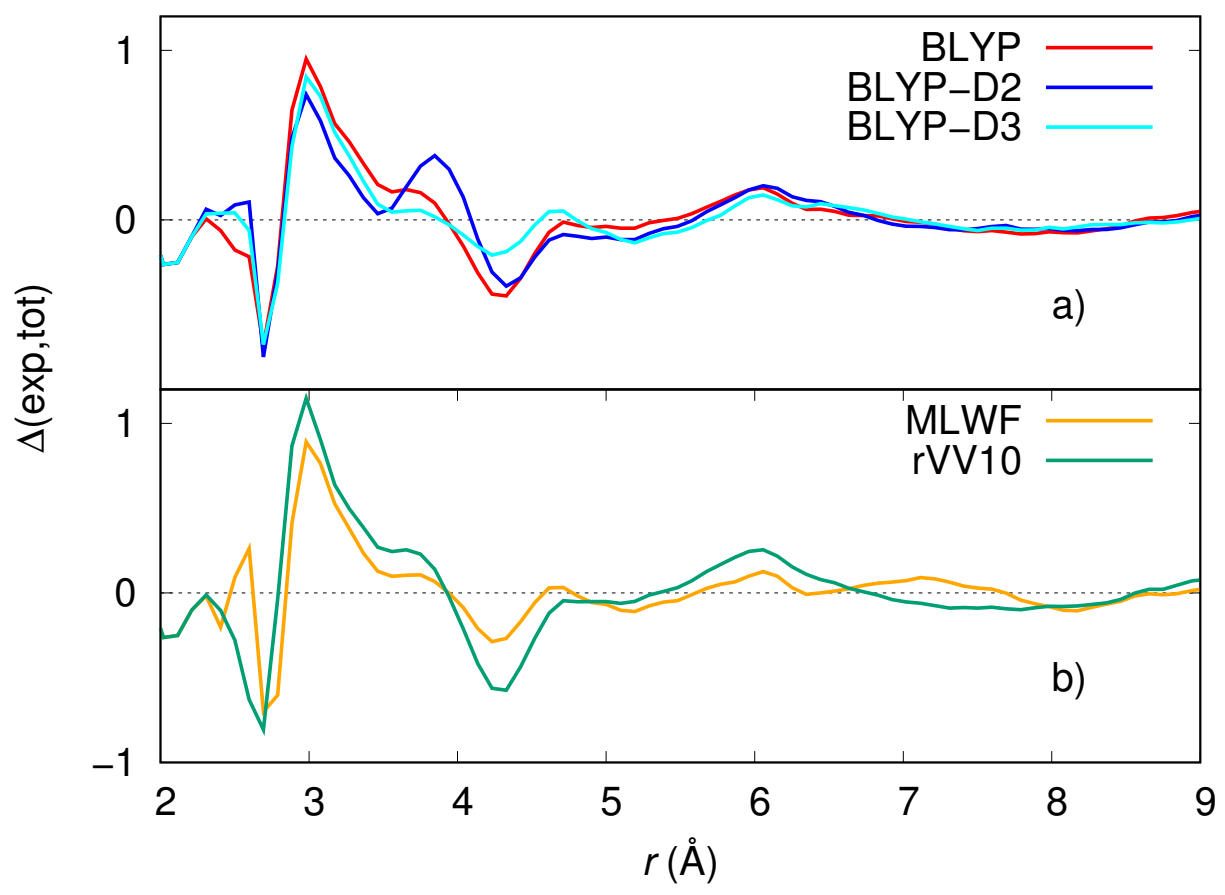

Figure 2: $g_{\mathrm{T}}(r)$. Difference functions obtained by subtracting the experimental points from each one of the calculated total pair correlation function. Experiments from Ref. 36.

This analysis is based on the assumption that the experimental curve is the one to be employed when assessing the reliability of the different strategies to account for vdW contributions. Globally, it underscores the legitimacy of schemes correcting Kohn-Sham recipes via the use of dispersion formulas $\left(\mathrm{vdW}_{\mathrm{D} 3}, \mathrm{vdW}_{\mathrm{W}}\right)$ either highly refined (the first) as a posteriori addition to the total energy or (the second) adapting to the dynamical evolution of the electronic structure.

An additional method to analyze our results consists in taking as reference data those obtained within the BLYP scheme with no account of dispersion forces. The situation is summarized in Table 3 again by taking advantage of the integrals of the absolute value of $\Delta$ (NovdW, tot), this function being visualized in Fig. 3 .

Results obtained via vdW $\mathrm{VV}, \mathrm{rVV} 10$ are by far the closest to NovdW, followed by $\mathrm{vdW}_{\mathrm{D} 2}$. However, $\mathrm{vdW}_{\mathrm{W}}$ behaves in a way not too dissimilar. This proves that this scheme is capable of ensuring a twofold effect. On the one hand, it improves upon BLYP approaching the experimental results. On the other hand, this occurs without departing drastically from the same BLYP data 


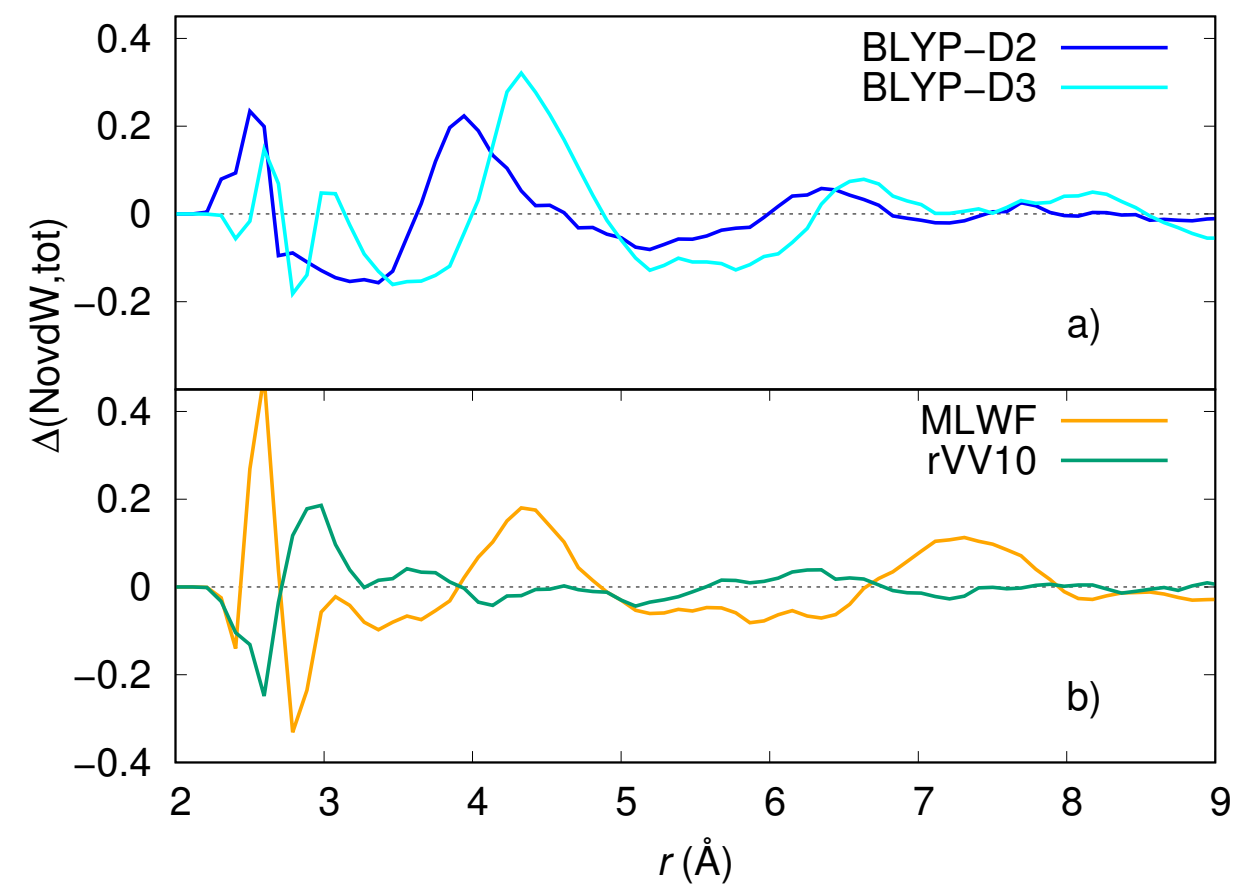

Figure 3: $g_{\mathrm{T}}(r)$. Difference functions obtained by subtracting the BLYP (NovdW) points from each one of the other available (calculated) total pair correlation functions

Table 3: Integral of the absolute value of the differences between a given set of data of $g_{\mathrm{T}}(r)$ and the BLYP (NovdW) data on the range [3-9] $\AA$.

\begin{tabular}{c|c} 
Functional & $\mathrm{I}_{\mid \Delta(\text { NovdW,tot }) \mid}$ (a. u.) \\
\hline rVV10 $\left(\mathrm{vdW}_{\mathrm{vv}}\right)$ & 0.175 \\
BLYP-D2 $\left(\mathrm{vdW}_{\mathrm{D} 2}\right)$ & 0.515 \\
BLYP-MLWF $\left(\mathrm{vdW}_{\mathrm{W}}\right)$ & 0.684 \\
BLYP-D3 $\left(\mathrm{vdW}_{\mathrm{D} 3}\right)$ & 0.942
\end{tabular}

in a way that could be interpreted as an artificial effect overestimating the actual correction due to dispersion forces. This is exactly the pitfall invoked in our previous study ${ }^{10}$ when comparing the performance of schemes depending or not depending explicitly on changes of the dispersion coefficients during the time evolution.

Therefore, it appears that the schemes employed can be grossly classified in two categories, i.e. those reducing the gap between the experimental data and the NovdW solution( $\left.\operatorname{vdW}_{\mathrm{D} 3}, \mathrm{vdW}_{\mathrm{W}}\right)$ and those closer to NovdW than to the experimental data ( $\mathrm{vdW}_{\mathrm{VV}}$ and to a smaller extent $\left.\mathrm{vdW}_{\mathrm{D} 2}\right)$. However, $\mathrm{vdW}_{\mathrm{W}}$ stands as the best compromise between these two tendencies, under the assump- 
tion that for this specific system one expects both corrections with respect to the mere BLYP NovdW data and a better agreement with experiments.

\subsection{Partial pair correlation function}

In the absence of experimental data for the partial pair correlation functions it is instructive to employ them to complete the analysis relative to the performances of the different schemes when compared to BLYP NovdW (Fig. 4, 6 and 5).

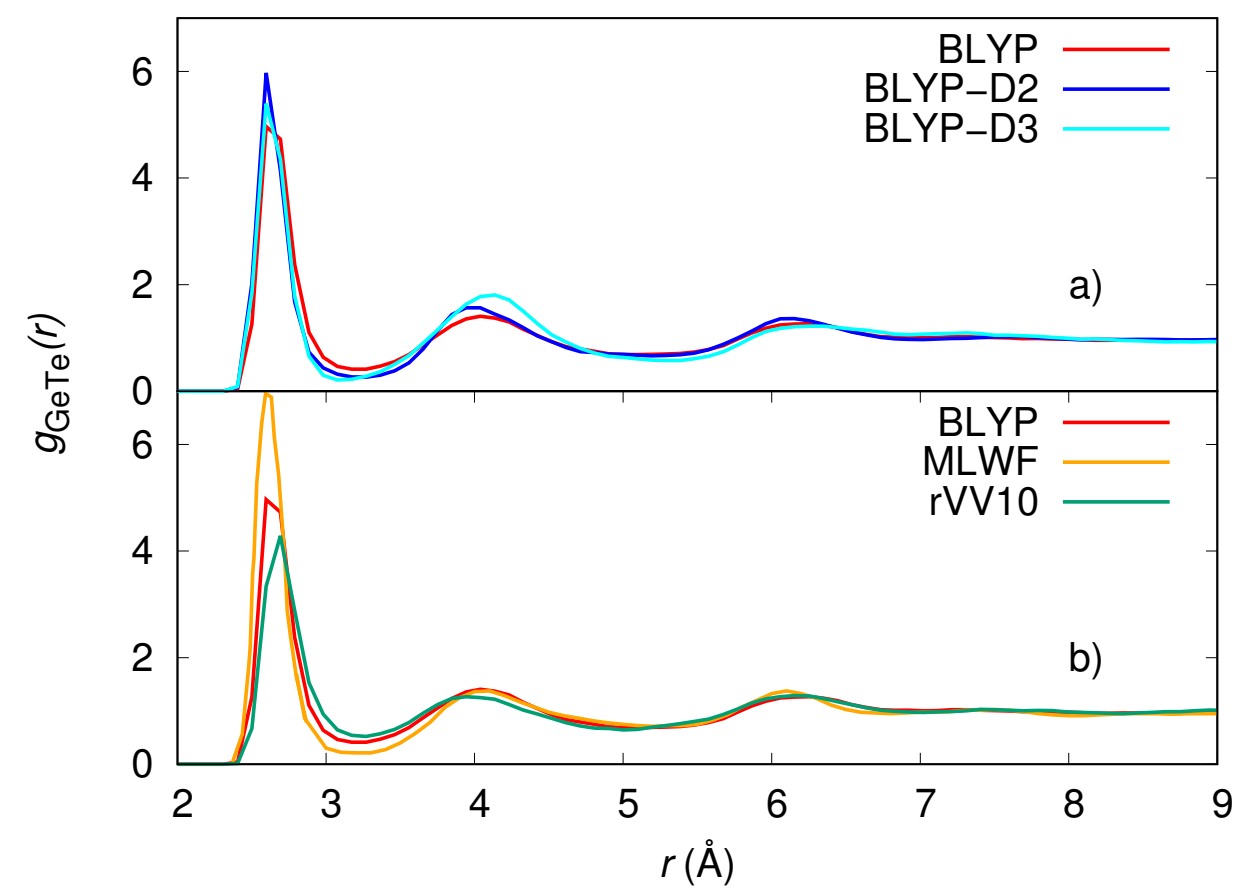

Figure 4: GeTe partial pair correlation functions.

In the case of $g_{\mathrm{GeTe}}(r)$ the five different profiles shown in Fig. 4 are quite similar, with vdW $\mathrm{D}_{\mathrm{D} 3}$ mostly departing from NovdW for $r>3 \AA$. This is confirmed by the data on the integrals of the absolute value of $\Delta(\mathrm{NovdW}, \mathrm{GeTe})\left(\right.$ Table 4). The pair correlation function $g_{\mathrm{TeTe}}(r)$ (Fig. 5) is at very origin of the close behavior of NovdW and $\mathrm{vdW}_{\mathrm{VV}}$ mentioned when considering $g_{\mathrm{T}}(r)$, keeping in mind that this partial pair correlation function bears the largest contribution in the weighted sum of partials leading to $g_{\mathrm{T}}(r)$. For $\mathrm{vdW}_{\mathrm{VV}}$ the departure from NovdW is very limited 


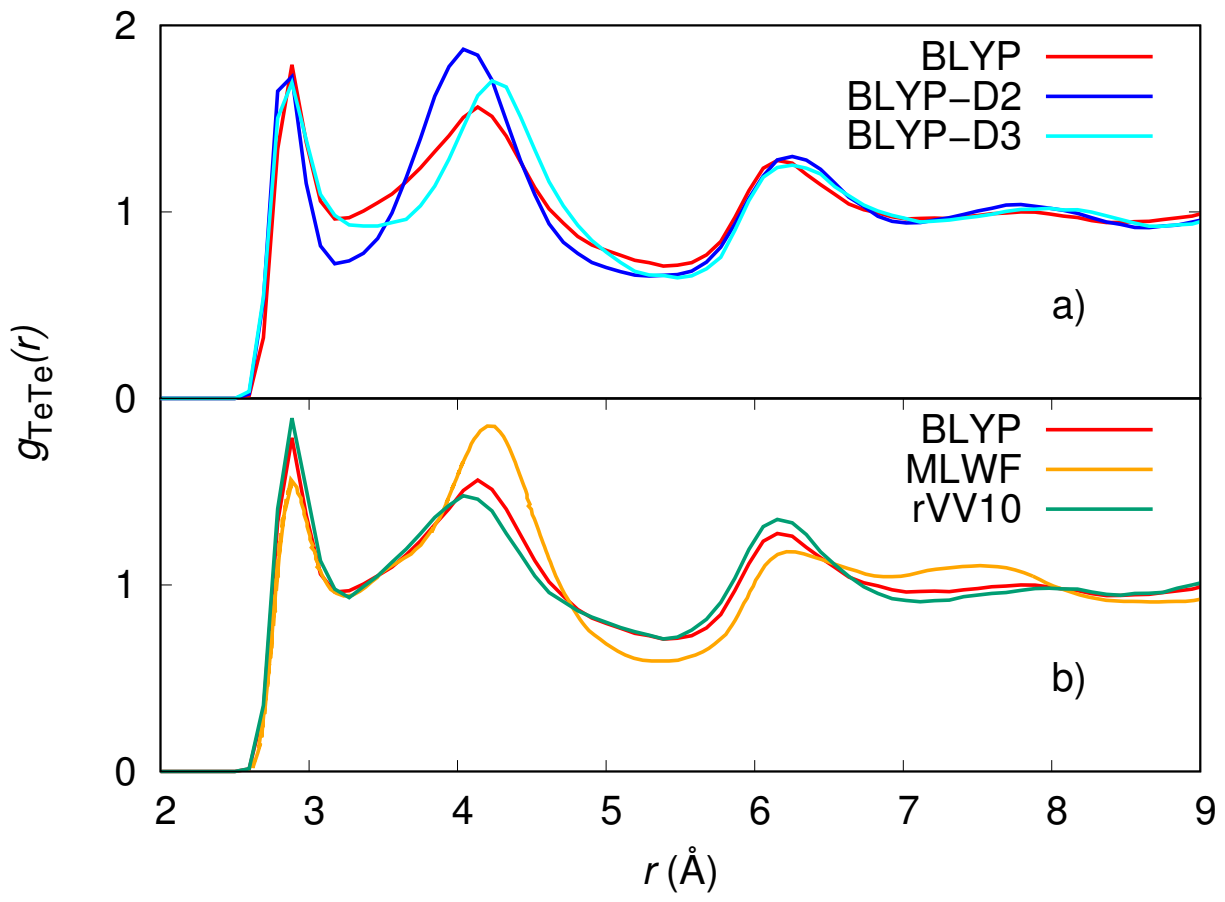

Figure 5: TeTe partial pair correlation functions.

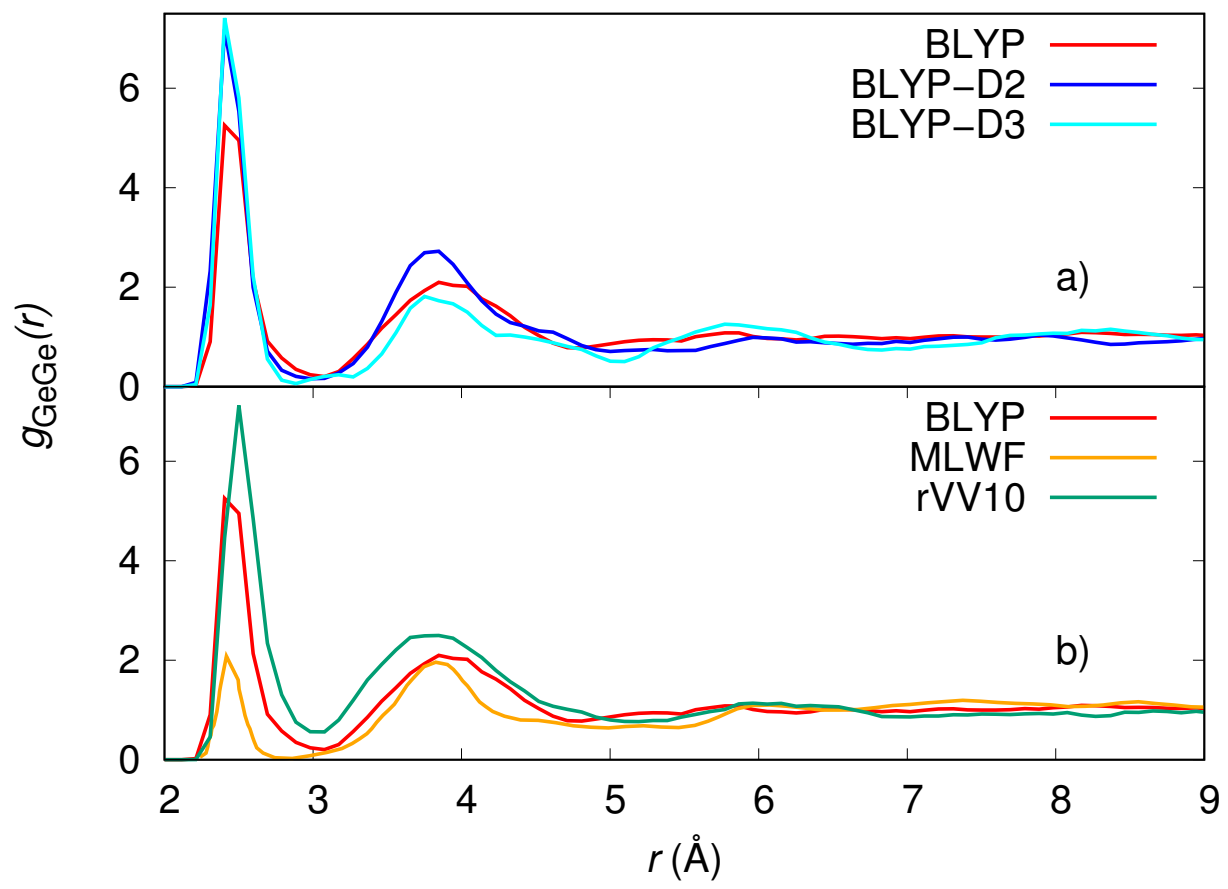

Figure 6: GeGe partial pair correlation functions. 
Table 4: Integral of the absolute value of the differences between a given set of data of partial pair correlation functions and the BLYP (NovdW) data on the range [3-9] $\AA$.

\begin{tabular}{c|c|c|c} 
Functional & $\mathrm{I}_{\mid \Delta(\text { NovdW,GeTe) } \mid}$ (a. u.) & $\mathrm{I}_{\mid \Delta(\text { NovdW,TeTe }) \mid}$ (a. u.) & $\mathrm{I}_{\mid \Delta(\text { NovdW,GeGe) } \mid}$ (a. u.) \\
\hline BLYP-D2 (vdW & 0.617 & 1.784 \\
BLYP-D3 (vdW $\left._{D 3}\right)$ & 0.914 & 1.107 & 3.599 \\
BLYP-MLWF (vdW & 0.673 & 0.993 & 2.210 \\
rVV10 (vdW & 0.429 & 0.227 & 1.751
\end{tabular}

in the range considered, with the integral of the absolute value of the corresponding difference function $\Delta$ (NovdW, TeTe) taking the smallest value among the twelve available values in Table 4. The case of $g_{\mathrm{GeGe}}(r)$ is the one featuring the largest discrepancies between BLYP NovdW and the other schemes (see Fig. 6) and yet is the one with the smallest impact on $g_{\mathrm{T}}(r)$. Regardless of the behavior in the region of the first peak, one notices that the height of the second peak is overestimated $\left(\mathrm{vdW}_{\mathrm{VV}}, \mathrm{vdW}_{\mathrm{D} 2}\right)$ or underestimated $\left(\mathrm{vdW}_{\mathrm{W}}, \mathrm{vdW}_{\mathrm{D} 3}\right)$, while for $r>4 \AA$ once again $\mathrm{vdW}_{\mathrm{VV}}$ and $\mathrm{vdW}_{\mathrm{D} 2}$ are those closer to NovdW. We conclude that the decomposition in the three partial structure factors helps confirming that there are sizeable differences among the four different vdW schemes employed in this work to include the dispersion forces. Of particular interest is the close behavior of NovdW and $\mathrm{vdW}_{\mathrm{VV}}$ data for Te-Te correlations, keeping in mind that $\mathrm{vdW}_{\mathrm{VV}}$ is the only scheme employed including the dispersion contribution directly in the energy functional.

\section{Conclusion}

Assessing the performances of theoretical recipes for the dispersion forces in a specific case is a valuable objective in the search of quantitative atomic-scale descriptions. This has been stressed by recent contribution in the area of DFT methodologies applied to the treatment of long range van der Waals interactions ${ }^{37-39}$.

Our selection of glassy $\mathrm{GeTe}_{4}$ proved worthwhile since this system is highly sensitive to the kind of vdW schemes employed although none of them brings crucial improvements upon the original DFT-BLYP approach devoid of dispersion contributions. The present work is the third of a series devoted to the structure of glassy $\mathrm{GeTe}_{4}$ and to the relationship between the structural features, 
expressed via the pair correlation functions, and the account of dispersion forces. In the first paper, we came to the conclusion that the atomic structure does depend on the choice of the exchangecorrelation functional and, to a minor extent, to the account of dispersion forces ${ }^{6}$. In the second, we considered a scheme accounting explicitly for changes in time of the van der Waals coefficient (Maximally Localized Wannier Functions method) ${ }^{10}$. Here we aimed at a more complete analysis of the effect of dispersion forces by relying on four different methods and on large set of equilibrium FPMD trajectories. Overall, we were able to demonstrate that the best agreement with experiments in terms of total pair correlation function is obtained by using the D3 or the MLWF schemes. This is the most straightforward conclusion one can come up with in terms of an objective analysis of our data. The good performance of the D3 scheme is related to its less empirical character. In fact, in the D3 method the specific molecular environment is considered by using atom-pair specific $C_{6}$ coefficients which include local information in the form of geometrymotivated, fractional coordinated numbers. For this reason, D3 is certainly closer in spirit to the MLWF method than its precursor D2.

In view of the expected non negligible and yet limited impact of the dispersion forces on our specific system, we have also paid attention to the performances of the different recipes in terms of comparison with the bare BLYP results. In this case, the best result is given by the rVV10 scheme, i.e. a functional including dispersion forces via a non-local construction in which there is no analytical separation from the energy functional. Consideration of both criteria (better agreement with experiments and moderate departure from the original BLYP data with no dispersion) led us to conclude that the MLWF scheme is the best compromise for situations in which expected changes due to dispersion forces are deemed to be of limited extent. These considerations are confirmed by the analysis of the partial pair correlation functions. In summary, this work clearly points out that the assessment of schemes for the account of the dispersion forces is not necessarily univocal, although the search of a better comparison with experiments might always appear as a primary legitimate objective. Our extended analysis and the use of different approaches contribute to a better understanding of a very subtle and controversial issue fostering a longstanding debate on the 
role and the impact of dispersion forces. The results obtained will prove especially important to better control bonding situations where the weight of the dispersion forces is smaller in comparison to other dominant bonding contributions.

\section{Acknowledgment}

Calculations were performed by using resources from GENCI (Grand Equipement National de Calcul Intensif) under Grants No. DARI-A0080906092, DARI-0910296 and DARI-095071, and Pôle HPC Equipex Equip@Meso project (Programme Investissements d'Avenir) and the CPER Alsacalcul/Big Data of the University of Strasbourg, and clustphy2 at the IEMN.

\section{References}

(1) Huang, C.-C.; Hewak, D.; Badding, J. Deposition and characterization of germanium sulphide glass planar waveguides. Opt. Express 2004, 12, 2501-2506.

(2) Kato, N.; Fukano, T.; Takeda, Y.; Takeichi, A.; Motohiro, T.; Kawai, S. GeS $2 /$ metal thin film bilayered structures as write-once-type optical recording materials. J. Appl. Phys. 2006, 100, 113115.

(3) Wuttig, M.; Yamada, N. Phase-Change Materials for Rewriteable Data Storage. Nature Mater. 2007, 6, 824-832.

(4) Bouzid, A.; Ori, G.; Boero, M.; Lampin, E.; Massobrio, C. Atomic-scale structure of the glassy $\mathrm{Ge}_{2} \mathrm{Sb}_{2} \mathrm{Te}_{5}$ phase change material: A quantitative assessment via first-principles molecular dynamics. Phys. Rev. B 2017, 96, 224204.

(5) Bouzid, A.; Gabardi, S.; Massobrio, C.; Boero, M.; Bernasconi, M. First-Principles Study of Amorphous $\mathrm{Ga}_{4} \mathrm{Sb}_{6} \mathrm{Te}_{3}$ Phase-Change Alloys. Phys. Rev. B 2015, 91, 184201. 
(6) Bouzid, A.; Massobrio, C.; Boero, M.; Ori, G.; Sykina, K.; Furet, E. Role of the van Der Waals Interactions and Impact of the Exchange-Correlation Functional in Determining the Structure of Glassy GeTe 4 . Phys. Rev. B 2015, 92, 134208.

(7) Becke, A. D. Density-functional exchange-energy approximation with correct asymptotic behavior. Phys. Rev. A 1988, 38, 3098-3100.

(8) Lee, C.; Yang, W.; Parr, R. G. Development of the Colle-Salvetti correlation-energy formula into a functional of the electron density. Phys. Rev. B 1988, 37, 785-789.

(9) Grimme, S. Semiempirical GGA-type density functional constructed with a long-range dispersion correction. J. Comput. Chem. 2006, 27, 1787-1799.

(10) Massobrio, C.; Martin, E.; Chaker, Z.; Boero, M.; Bouzid, A.; Le Roux, S.; Ori, G. Sensitivity to Dispersion Forces in First-Principles Modeling of Disordered Chalcogenides. Front. in Mat. 2018, 5, 78 .

(11) Car, R.; Parrinello, M. Unified approach for molecular dynamics and density-functional theory. Phys. Rev. Lett. 1985, 55, 2471-2474.

(12) Wannier, G. H. The structure of electronic excitation levels in insulating crystals. Phys. Rev 1937, 52, 191-197.

(13) Marzari, N.; Vanderbilt, D. Maximally-localized generalized Wannier functions for composite energy bands. Phys. Rev. B 1997, 56, 12847-12865.

(14) Silvestrelli, P. L.; Marzari, N.; Vanderbilt, D.; Parrinello, M. Maximally-localized Wannier functions for disordered systems: Application to amorphous silicon. Solid State Commun. 1998, 107, 7-11.

(15) Ikeda, T.; Boero, M. Role of van der Waals corrections in first principles simulations of alkali metal ions in aqueous solutions. J. Chem. Phys. 2015, 143, 194510. 
(16) Hutter, J.; Parrinello, M.; Marx, D.; Focher, P.; Tuckerman, M.; Andreoni, W.; Curioni, A.; Fois, E.; Rothlisberger, U.; Giannozzi, P. Computer code CPMD. IBM Zurich Research Laboratory and MPI für Festkörperforschung 1995, 2001.

(17) Bouzid, A.; Le Roux, S.; Ori, G.; Boero, M.; Massobrio, C. Origin of structural analogies and differences between the atomic structures of $\mathrm{GeSe}_{4}$ and $\mathrm{GeS}_{4}$ glasses: A first principles study. J. Chem. Phys. 2015, 143, 034504.

(18) Lampin, E.; Bouzid, A.; Ori, G.; Boero, M.; Massobrio, C. Impact of dispersion forces on the atomic structure of a prototypical network-forming disordered system: The case of liquid $\mathrm{GeSe}_{2}$. J. Chem. Phys. 2017, 147, 044504.

(19) Nosé, S. A unified formulation of the constant temperature molecular dynamics methods. $J$. Chem. Phys. 1984, 81, 511-519.

(20) Hoover, W. G. Canonical dynamics: equilibrium phase-space distributions. Phys. Rev. A 1985, 31, 1695-1697.

(21) Blöchl, P. E.; Parrinello, M. Adiabaticity in first-principles molecular dynamics. Phys. Rev. B 1992, 45, 9413-9416.

(22) Micoulaut, M.; Vuilleumier, R.; Massobrio, C. Improved modeling of liquid GeSe 2 : Impact of the exchange-correlation functional. Phys. Rev. B 2009, 79, 214205.

(23) Giacomazzi, L.; Massobrio, C.; Pasquarello, A. Vibrational properties of vitreous GeSe2with the Becke-Lee-Yang-Parr density functional. Journal of Physics: Condensed Matter 2011, $23,295401$.

(24) Perdew, J. P.; Wang, Y. Accurate and simple analytic representation of the electron-gas correlation energy. Phys. Rev. B 1992, 45, 13244-13249.

(25) Andersson, Y.; Langreth, D. C.; Lundqvist, B. I. van der Waals interactions in DensityFunctional Theory. Phys. Rev. Lett. 1996, 76, 102-105. 
(26) Resta, R. Macroscopic Electric Polarization as a Geometric Quantum Phase. Europhysics Letters (EPL) 1993, 22, 133-138.

(27) Vanderbilt, D.; King-Smith, R. D. Electric polarization as a bulk quantity and its relation to surface charge. Phys. Rev. B 1993, 48, 4442-4455.

(28) Silvestrelli, P. L.; Ambrosetti, A. van der Waals interactions in DFT using Wannier functions without empirical parameters. The Journal of Chemical Physics 2019, 150, 164109.

(29) Giannozzi, P.; Baroni, S.; Bonini, N.; Calandra, M.; Car, R.; Cavazzoni, C.; Ceresoli, D.; Chiarotti, G. L.; Cococcioni, M.; Dabo, I.; et al., QUANTUM ESPRESSO: a modular and open-source software project for quantum simulations of materials. J. Phys.: Cond. Mat. 2009, 21, 395502.

(30) Giannozzi, P.; Andreussi, O.; Brumme, T.; Bunau, O.; Nardelli, M. B.; Calandra, M.; Car, R.; Cavazzoni, C.; Ceresoli, D.; Cococcioni, M.; et al., Advanced capabilities for materials modelling with QUANTUM ESPRESSO. J. Phys.: Cond. Mat. 2017, 29, 465901.

(31) Giannozzi, P.; Baseggio, O.; Bonfà, P.; Brunato, D.; Car, R.; Carnimeo, I.; Cavazzoni, C.; de Gironcoli, S.; Delugas, P.; Ferrari Ruffino, F.; et al., Quantum ESPRESSO toward the exascale. J. Chem. Phys. 2020, 152, 154105.

(32) Grimme, S.; Antony, J.; Ehrlich, S.; Krieg, H. A consistent and accurate ab initio parametrization of density functional dispersion correction (DFT-D) for the 94 elements H-Pu. J. Chem. Phys. 2010, 132, 154104.

(33) Stohr, M.; Van Voorhis, T.; Tkatchenko, A. Theory and practice of modeling van der Waals interactions in electronic-structure calculations. Chem. Soc. Rev. 2019, 48, 4118-4154.

(34) Sabatini, R.; Gorni, T.; de Gironcoli, S. Nonlocal van der Waals density functional made simple and efficient. Phys. Rev. B 2013, 87, 041108. 
(35) Vydrov, O. A.; Van Voorhis, T. Nonlocal van der Waals density functional: The simpler the better. J. Chem. Phys. 2010, 133, 244103.

(36) Kaban, I.; Halm, T.; Hoyer, W.; Jovari, P.; Neuefeind, J. Short-range order in amorphous germanium-tellurium alloys. J. Non-Cryst. Solids 2003, 326, 120-124.

(37) Raty, J. Y.; Zhang, W.; Luckas, J.; Mazzarello, R.; Bichara, C.; Wuttig, M. Aging mechanisms in amorphous phase-change materials. Nat. Comm. 2015, 6, 7467.

(38) Lee, K.; Murray, E. D.; Kong, L.; Lundqvist, B. I.; Langreth, D. C. Higher-accuracy van der Waals density functional. Phys. Rev. B 2010, 82, 081101.

(39) Tkatchenko, A.; Scheffler, M. Accurate molecular van der Waals interactions from groundstate electron density and free-atom reference data. Phys. Rev. Lett. 2009, 102, 073005.

\section{Supplementary material}

The data that support the findings of this study are available from the corresponding author upon request. 


\section{Graphical TOC Entry}

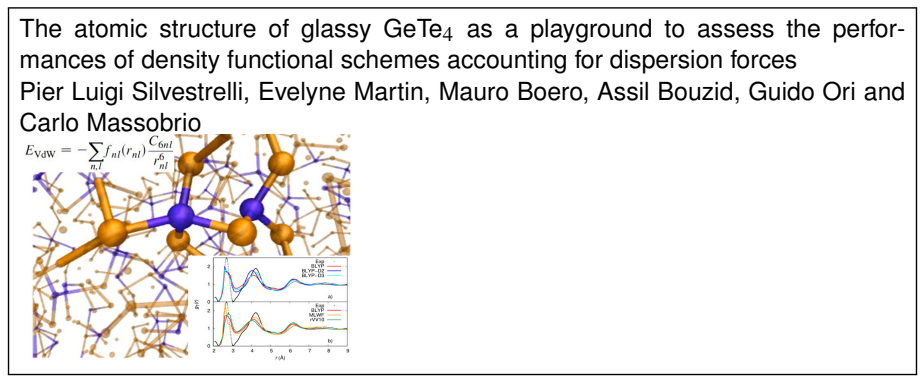

